\title{
Cloud Atlas: Weak Color Modulations Due to Rotation in the Planetary-mass Companion GU Psc b and 11 Other Brown Dwarfs
}

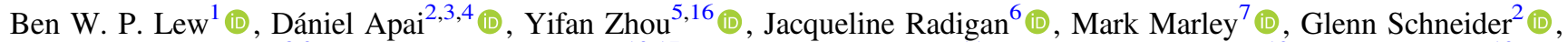

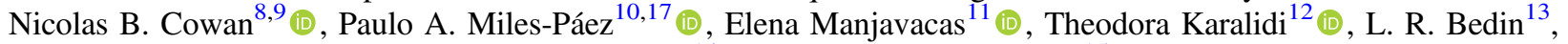 \\ Patrick J. Lowrance ${ }^{14}$ (D), and Adam J. Burgasser ${ }^{15}$ (D) \\ ${ }^{1}$ Lunar and Planetary Laboratory, The University of Arizona, 1640 E. University Blvd.,Tucson, AZ 85718, USA; weipenglew@ email.arizona.edu \\ ${ }^{2}$ Department of Astronomy and Steward Observatory, The University of Arizona, 933 N. Cherry Ave., Tucson, AZ, 85721, USA \\ ${ }^{3}$ Lunar and Planetary Laboratory, The University of Arizona, 1640 E. University Blvd., Tucson, AZ 85718, USA \\ ${ }^{4}$ Earths in Other Solar Systems Team, NASA Nexus for Exoplanet System Science, USA \\ ${ }^{5}$ Department of Astronomy, University of Texas, Austin, TX 78712, USA \\ ${ }^{6}$ Utah Valley University, 800 West University Parkway, Orem, UT 84058, USA \\ ${ }_{8}^{7}$ NASA Ames Research Center, Naval Air Station, Moffett Field, Mountain View, CA 94035, USA \\ ${ }^{8}$ Department of Physics, McGill University, 3600 rue University, Montréal, QC, H3A 2T8, Canada \\ ${ }^{9}$ Department of Earth \& Planetary Sciences, McGill University, 3450 rue University, Montréal, QC, H3A 0E8, Canada \\ ${ }^{10}$ European Southern Observatory, Karl-Schwarzschild-Straße 2, D-85748 Garching, Germany \\ ${ }^{11}$ W.M. Keck Observatory, Mamalahoa Hwy, Kamuela, HI 96743, USA \\ ${ }^{12}$ Department of Physics, University of Central Florida, 4000 Central Florida Boulevard, Orlando, FL 32816, USA \\ ${ }^{13}$ INAF-Osservatorio Astronomico di Padova, Vicolo dell'Osservatorio 5, I-35122 Padova, Italy \\ ${ }^{14}$ IPAC-Spitzer, MC 314-6, California Institute of Technology, Pasadena, CA 91125, USA \\ ${ }^{15}$ Astrophysics and Space Science, University of California San Diego, La Jolla, CA 92093, USA \\ Received 2019 October 13; revised 2019 December 1; accepted 2019 December 4; published 2020 February 25
}

\begin{abstract}
Among the greatest challenges in understanding ultracool brown dwarf and exoplanet atmospheres is the evolution of cloud structure as a function of temperature and gravity. In this study, we present the rotational modulations of GU Psc b-a rare mid-T spectral type planetary-mass companion at the end of the L/T spectral type transition. Based on the Hubble Space Telescope/WFC3 1.1-1.67 $\mu \mathrm{m}$ time-series spectra, we observe a quasi-sinusoidal light curve with a peak-to-trough flux variation of $2.7 \%$ and a minimum period of $8 \mathrm{~h}$. The rotation-modulated spectral variations are weakly wavelength-dependent, or largely gray between 1.1 and $1.67 \mu \mathrm{m}$. The gray modulations indicate that heterogeneous clouds are present in the photosphere of this low-gravity mid-T dwarf. We place the color and brightness variations of GU Psc $b$ in the context of rotational modulations reported for mid-L to late-T dwarfs. Based on these observations, we report a tentative trend: mid-to-late $\mathrm{T}$ dwarfs become slightly redder in $J-H$ color with increasing $J$-band brightness, while L dwarfs become slightly bluer with increasing brightness. If this trend is verified with more T-dwarf samples, it suggests that in addition to the mostly gray modulations, there is a second-order spectral-type dependence on the nature of rotational modulations.
\end{abstract}

Unified Astronomy Thesaurus concepts: Brown dwarfs (185); Exoplanet atmospheres (487); Planetary atmospheres (1244); Exoplanet atmospheric variability (2020); T dwarfs (1679)

\section{Introduction}

One of the most perplexing observations of the ultracool atmospheres of brown dwarfs and directly imaged exoplanets is the prominent color evolution across the $\mathrm{L} / \mathrm{T}$ spectral type transition. Over a narrow temperature range $(\sim 100 \mathrm{~K})$ the atmospheres transition from red (in the near-infrared, $J-H \sim 1.3)$ to blue $(J-H \sim 0.0)$ colors. It has been proposed that this color evolution could be caused by cloud thinning (Ackerman \& Marley 2001; Saumon \& Marley 2008), cloud patchiness (Burgasser et al. 2002; Marley et al. 2010), cloud structure evolution (Tsuji \& Nakajima 2003; Burrows et al. 2006; Charnay et al. 2018), and possibly even $\mathrm{CO} / \mathrm{CH}_{4}$ compositional-gradient-driven instability (Tremblin et al. 2016, 2019). Brown dwarfs with mid-T spectral types also tend to be about 0.5 magnitudes brighter in the $J$ band than earlier and later spectral type counterparts-also known as the $J$-band brightening, possibly as a result of cloud disruption (Burgasser et al. 2002, 2006b; Dupuy \& Liu 2012). While

\footnotetext{
${ }^{16}$ Harlan J. Smith McDonald Observatory Postdoctoral Fellow.

17 ESO Fellow.
}

models of ultracool atmospheres had considerable success in fitting the $\mathrm{L}$ spectral type sequence (with thick silicate condensate clouds) and the late-T spectral type brown dwarfs (with mostly cloud-free atmospheres), the rapid color evolution and brightening across the $\mathrm{L} / \mathrm{T}$ transition point to the existence of processes that are not well understood.

Therefore, understanding atmospheric and cloud evolution from late-L spectral types though the $\mathrm{L} / \mathrm{T}$ transition to late- $\mathrm{T}$ dwarfs remains an important challenge. It is clear that the color changes carry important information about the nature of the processes that occur in these cooling atmospheres.

Surface gravity may be part of the puzzle too. Small samples of $\mathrm{L} / \mathrm{T}$ transition brown dwarfs suggest that the $\mathrm{L} / \mathrm{T}$ transition occurs at lower effective temperatures for low-gravity objects (Metchev \& Hillenbrand 2006; Dupuy et al. 2009; Marley et al. 2012; Bowler et al. 2013; Liu et al. 2016; Miles-Páez et al. 2017). The magnitude of $J$-band brightening could also be larger for low-gravity objects if we include directly imaged planets (see Figure 16 in Dupuy \& Liu 2012). Alas, as cooling objects cross swiftly the $\mathrm{L} / \mathrm{T}$ transition, very few brown dwarfs are known with low masses at the $\mathrm{L} / \mathrm{T}$ transition, making it 
difficult to test model predictions about the interplay of surface gravity and cloud evolution.

A particularly important probe of atmospheric properties is time-resolved high-precision (subpercent level) spectrophotometry, that can-through the rotation of the target-explore nonuniform brightness distribution in an atmosphere with fixed gravity and interior temperature. Such rotational mapping studies have been used successfully to constrain cloud properties in ultracool atmospheres, including those with planetary masses (e.g., Apai et al. 2013; Buenzli et al. 2014; Metchev et al. 2015; Zhou et al. 2016, 2019; Apai et al. 2017; Biller 2017; Manjavacas et al. 2019; Miles-Páez et al. 2019). The wavelength dependence of rotation modulations sheds light on the variations of cloud particle sizes, molecular abundances, and photospheric temperatures. In a rotating atmosphere, the temporal modulations at a given wavelength probe the atmosphere's spatial structure in a pressure range. Consequently, comparisons of the modulations observed at different wavelengths probe pressure-dependent properties in the atmosphere.

Multiple studies have used time-resolved ground-based photometry (e.g., Artigau et al. 2009; Radigan et al. 2012; Biller et al. 2013; Radigan 2014), spectroscopy (e.g., Schlawin et al. 2017), or space-based spectrophotometry (e.g., Buenzli et al. 2012, 2015; Yang et al. 2015, 2016; Karalidi et al. 2016; Lew et al. 2016) to explore the variations of the near-infrared (near-IR) colors of rotating brown dwarfs (from mid-L to T8 spectral types). All of these studies found gray, i.e., only weakly wavelength-dependent modulations in the near-IR, even for objects with large-amplitude $(>10 \%)$ modulations. Radiative-transfer-based models presented in Apai et al. (2013) explain the gray modulations with a correlated change in effective temperature and cloud thickness; this study and that of Radigan et al. (2012) show that changes in a single model parameter (temperature or cloud thickness) cannot explain the observed modulations. Similar conclusions have been drawn by Biller et al. (2018), who observed the planetary-mass late-L dwarf PSO J318.5338-22.8603. They suggest that the heterogeneous high-altitude clouds or extended silicate clouds could explain the weak wavelength-dependence of near-IR and midIR modulation amplitudes, as well as the phase offset between the near-IR and mid-IR light curves.

These examples demonstrate the power of time-resolved highprecision spectrophotometry in constraining the heterogeneous cloud properties of individual atmospheres and-through comparisons of objects spanning the $\mathrm{L}-\mathrm{L} / \mathrm{T}-\mathrm{T}$ sequence- the potential for deciphering cloud evolution in cooling atmospheres. For a more complete discussion of results from timeresolved studies we refer to recent reviews (Biller 2017; Artigau 2018).

In this paper we present a new, space-based, and highprecision time-resolved spectrophotometric study of GU Psc b, one of the rare planetary-mass brown dwarfs at the end of the $\mathrm{L} / \mathrm{T}$ sequence. In Section 2 and 3 we described the target, the observations, and data reduction process. We present the spectra and light curves in Section 4. In Section 5, we describe the compilation of published data and the analysis of the colormagnitude variations of 12 brown dwarfs. We discuss the implications of our results to the color change in the $\mathrm{L} / \mathrm{T}$ transition in Section 6 and summarize our conclusions in Section 7.

\section{GU Psc and GU Psc b}

GU Psc (or 2MASS J01123504+1703557) is an M3.5 dwarf at $47.6 \mathrm{pc}$ based on the Gaia parallax of $21.00 \pm 0.07$ mas (Gaia Collaboration et al. 2016, 2018; Luri et al. 2018). Based on its kinematic and photometric properties, Malo et al. (2013) categorized the GU Psc system as a highly probable (96.9\%) member of the $\mathrm{AB}$ Doradus moving group (ABDMG), which is around $120 \pm 30 \mathrm{Myr}$ old (Zuckerman et al. 2004). The detection and measured width of $H \alpha$ emission of GU Psc by Riaz et al. (2006) indicates that the age of the system is in between 10 Myr-2 Gyr respectively (Barrado y Navascués \& Martín 2003; White \& Basri 2003; West et al. 2008). Naud et al. (2014, hereafter N14) find that the calculated X-ray luminosity $\left(\log \left(L_{x}\right)=29.1 \pm 0.3 \mathrm{erg} \mathrm{s}^{-1}\right.$ at $\left.48 \pm 5 \mathrm{pc}\right)$ of GU Psc based on the ROSAT observation is similar to that of other single $\mathrm{M}$ dwarfs in $\mathrm{ABDMG}$ and is higher than that of field stars. The kinematic properties, $\mathrm{H} \alpha$ emission, and X-ray luminosity measurements together suggest that GU Psc is a relatively young system compared to field dwarfs.

N14 provide an estimate of the GU Psc's metallicity $([\mathrm{Fe} / \mathrm{H}])$ that ranges from $-0.14 \pm 0.09$ to $0.1 \pm 0.13$ with various methods (Mann et al. 2013; Newton et al. 2014). The measured periodic variability of $1.0362 \pm 0.0005$ days (Norton et al. 2007) and $v \sin i\left(23 \mathrm{~km} \mathrm{~s}^{-1}\right.$; N14) of GU Psc suggest that it is a rapid rotator. A more detailed characterization of the GU Psc system can be found in N14.

GU Psc b, discovered by N14, is at a projected distance of $2000 \pm 200$ au from GU Psc. Based on the near-IR spectral index and the comparison with standard T dwarfs, N14 classify GU Psc b as a T3.5 \pm 1 dwarf. N14 find the best-fit effective temperature ranges from 1000 to $1100 \mathrm{~K}$ by comparing the near-IR spectrum of GU Psc b with atmospheric models (Baraffe et al. 2003; Saumon \& Marley 2008). Based on the ABDMG's age of $100 \pm 30 \mathrm{Myr}$ and the fitted effective temperature range, N14 estimate the mass of GU Psc b to be around 9-13 $M_{\mathrm{Jup}}$, which is close to the deuterium-burning mass limit of 12-13 $M_{\text {Jup }}$ (Saumon \& Marley 2008)commonly adopted as the borderline between brown dwarfs and giant planets with solar metallicity. Naud et al. (2017) report a tentative $J$-band photometric variability of GU Psc b with a peak-to-trough flux variation of $4 \pm 1 \%$ at a period of $5.9 \mathrm{hr}$ based on one out of three nights of 5-6 hr observation with the WIRCam Imager at the $3.6 \mathrm{~m}$ Canada-France-Hawaii Telescope.

\section{Observation and Data Reduction}

We utilized six consecutive Hubble Space Telescope (HST) orbits to observe the rotational modulation of GU Psc b in the G141 grism mode $(1.07-1.70 \mu \mathrm{m}$, spectral resolving power $\sim 130$ or dispersion of $4.7 \mathrm{~nm}^{\text {pixel }}{ }^{-1}$ with a plate scale of 0 ". 13 pixel $^{-1}$ ) of Wide Field Camera 3 (WFC3) on 2018 January 8. These observations are part of the HST Large Treasury Program Cloud Atlas (PI: Apai; ID: GO-14241). There are eleven $201 \mathrm{~s}$ long integration spectroscopic frames in each 96 minute long $H S T$ orbit. We restricted the orientation angle of $H S T$ to minimize possible spectral contamination from galaxies and bright stars within small angular separations from GU Psc b.

The data reduction pipeline is similar to that in Lew et al. (2016). In brief, our data reduction process started from flt. fits files, which are produced by the calwf 3 pipeline for 

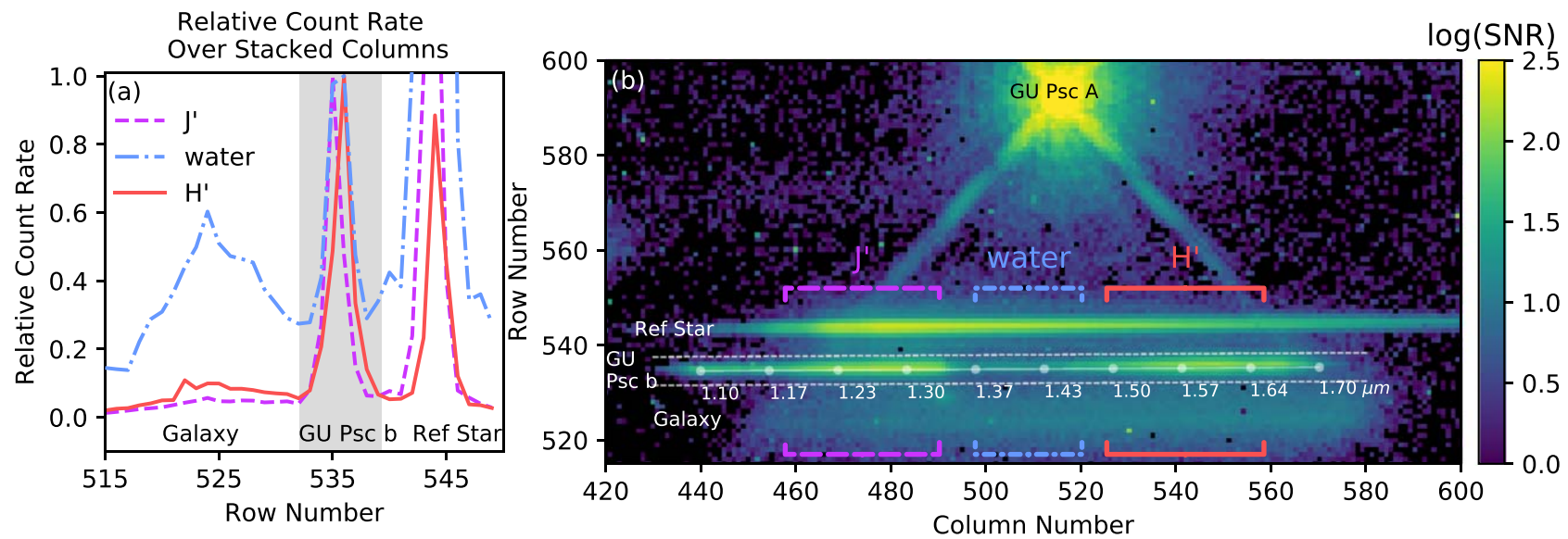

Figure 1. (a) Relative count rates of pixels stacked along the rows in panel (b) in three different wavelength regions: approximated $J$ band (dashed magenta line), water band (dashed-dotted blue line), and $H$ band (solid red line). In the spectral extraction region of GU Psc b, plotted in shaded gray, the flux contribution from nearby sources is lower than $\sim 10 \%$ for the $J^{\prime}$ and $H^{\prime}$ bands, but higher than $\sim 20 \%$ for the water band. (b) A cropped median image to illustrate the nearby reference star and galaxy spectra flanking that of GU Psc b. The image is color coded with signal-to-noise ratio in log scale. The three square brackets represent the $J^{\prime}$-, water-, and $H^{\prime}$-band regions in which the pixel count rates are stacked and summed along the rows. The dotted line with annotated wavelengths in microns is plotted for GU Psc b's spectrum. The two white dashed lines that bracket the dotted line mark the aperture (six pixels) for spectral extraction.

zero-read and bias calibration, dark image subtraction, flatfielding, detector nonlinearity correction, and cosmic-ray flagging. Our well-vetted pipeline (e.g., Buenzli et al. 2012; Apai et al. 2013; Yang et al. 2015; Zhou et al. 2018) then interpolated around the cosmic rays affected and other bad pixels before subtracting the sky background. We followed the method in Kümmel et al. (2011) for background reduction that scales the master sky image from Kümmel et al. (2011) for background reduction. We used Source Extractor (Bertin $\&$ Arnouts 1996) to extract the source location from the stacked direct images that are observed with the F132N filter at the beginning of each orbit for wavelength calibration. We used aXe (Kümmel et al. 2009) with a six-pixel-wide crossdispersion aperture for spectral extraction. We performed an absolute flux correction for finite aperture photometry by interpolating the table of encircled energy as a function wavelength and diameter of Table $6 \mathrm{~b}$ in Kuntschner et al. (2011).

We did not find a significant ramp effect (Smith et al. 2008; Long et al. 2014) in the six-orbit-long observation, therefore no ramp correction (e.g., Zhou et al. 2017) was performed. The less pronounced ramp effect in our data is likely because of the low count rate (peak count rate $<8 e^{-} \mathrm{s}^{-1}$ with an averaged count rate ${ }^{18}$ of $\left.\sim 2.8 e^{-} \mathrm{s}^{-1}\right)$ compared to that of the case studies in Zhou et al. (2017). Given a lower count rate, the ramp profile becomes more linear and is less significant compared to the photon noise. Any uncorrected ramp effect, which mostly increases the flux in the first orbit, will only increase the brightness variations reported in this study.

\subsection{Contamination Assessment}

As Figure 1 shows, there are two sources in close angular proximity of GU Psc b, leading to a second-order spectrum of a reference star and to a first-order spectrum of a galaxy (see also Figure 1 in N14). Also, there is a faint diffraction spike arises from the zeroth-order (undispersed) grism image of GU Psc.

\footnotetext{
18 The averaged count rate was calculated over a box that is six pixels wide in cross-dispersion direction and 130 pixels long in the spectral dispersion direction of GU Psc b.
}
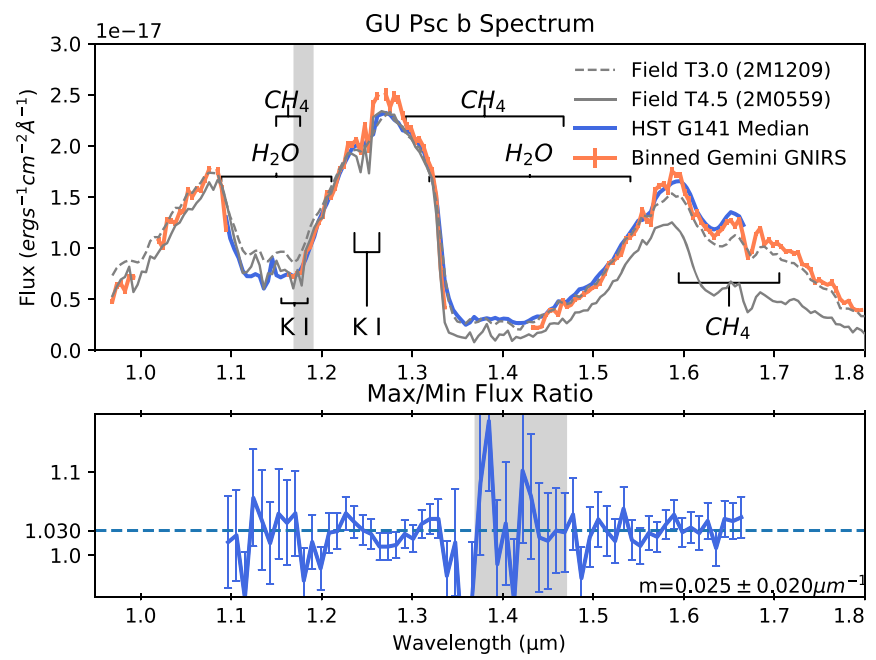

Figure 2. (a) Median-averaged spectra plotted in blue in comparison with the GNIRS spectrum in the solid orange line. Spectra of field T4.5 dwarf 2M0559 (solid gray line) and T3.5 2M1209 (dashed gray line), which are scaled to match the $J$-band maximum flux density of GU Psc b, are also plotted for comparison. The wavelength region in which the flux density is interpolated is in gray. (b) The maximum/minimum flux ratio among the six $H S T$ orbit averaged spectra suggests no strong wavelength dependence in the rotational modulations. The fitted wavelength-dependence slope $m$, excluding the water band (gray region), is shown at the bottom right.

The faint spike superimposes upon part of the spectrum of GU Psc b. To mitigate this, we interpolate the flux density in the $1.17-1.19 \mu \mathrm{m}$ region of the GU Psc b spectra (see Figure 2) to avoid possible contamination from GU Psc's zeroth-order diffraction spike.

To assess possible contamination from the two nearby sources, we sum the measured count rates in the same row $(x-$ axis) over three ranges of columns (i.e., the bracketed regions colored in magenta, blue, and red in Figure 1(b)), approximating the measured count rate in the $J^{\prime}(1.18-1.33 \mu \mathrm{m})$, water $(1.37-1.47 \mu \mathrm{m})$, and $H^{\prime}$ bands $(1.50-1.65 \mu \mathrm{m})$. The summed count rates are then plotted as a function of row number in Figure 1(a). In Figure 1(a), the summed count rate of GU Psc b 
spans roughly across rows 532-538 and is highlighted by the gray band. The gray region is similar to the aperture used for spectral extraction. ${ }^{19} \mathrm{We}$ normalize the summed count rate of the three ranges of columns so that the peak count rate of GU Psc equals one.

Figure 1(a) provides an order-of-magnitude estimation of the contamination level-within the spectral aperture for GU Psc b, the contamination level is less than $10 \%$ of the GU Psc b's peak count rate in the $J^{\prime}$ and $H^{\prime}$ bands, but much higher $(>20 \%)$ in the water band. A more sophisticated analysis that fits 1D three-Moffat profiles to the summed count rate from 1.1 to $1.7 \mu \mathrm{m}$ (column number 440-570) gives a similar estimate of the contamination level (13\%; see also Appendix A). Because of the low signal-to-noise ratio and the moderate level of contamination in the water band, in this study we choose to focus on the $J^{\prime}$ band, $H^{\prime}$ band, and the integrated white (1.1-1.67 $\mu \mathrm{m})$ light curve.

In Figure 3, the reference star's light curve shows a slight brightening trend at a subpercent level. A simple straight-line fit to the reference star's light curve gives a slope of $(9 \pm 2) \times 10^{-4} \mathrm{hr}^{-1}$, or $0.7 \%$ for an $8 \mathrm{hr}$ baseline. This linear-brightening trend is possibly an HST systematics that leads to visit-long slopes (e.g., Berta et al. 2012; Wakeford et al. 2016). In the field of view no other source's light curve has a similar signal-to-noise ratio to verify the presence of this possible subpercent-level systematics. The low signal-to-noise ratio light curve of the nearby background galaxy fluctuates at about $4 \%$ from the brightest to the dimmest state. The maximum contamination level of the flux variation from the nearby sources is roughly equal to the product of the flux contamination fraction and the flux variation of nearby sources, which is about a level of $13 \% \times 4 \%=0.52 \%$. The estimate of $0.52 \%$, which is a generous upper limit considering the different light curve profiles of between GU Psc b and other sources, is much lower than the observed peak-to-trough flux variation of $2.7 \%$ (see Section 4.2) in the integrated white light curve of GU Psc b.

We also check the distribution of flux density deviations from the median spectrum. We find only two data points with a $3.2 \sigma$ deviation from the median spectral flux densities among 124 spectral bins in 66 exposures. Therefore, no statistically significant deviation from the median spectrum is found assuming spectral points that are independent of each other. Therefore, our careful inspection of spectral variations and reduced images confirms that the observed rotational modulation is intrinsic to GU Psc b.

\section{Spectra and Rotational Modulations}

\subsection{Spectrum and Spectral Variations}

We plot the HST median spectra together with the Gemini Near-InfraRed Spectrograph (GNIRS) spectrum $(R \sim 800)$ from N14 in the top panel of Figure 2. We also overplot the spectra of two field T dwarfs, T4.5 2MASS J05591914 -1404488 (Burgasser et al. 2006a) and T3 2MASS J12095613-1004008 (Burgasser et al. 2004), which are normalized to the same $J$-band peak flux density as that of

\footnotetext{
19 The direction of spectral trace and the corresponding aperture for spectral extraction are not perfectly aligned with the $x$-axis of the image grid but with a field-dependent multi-order polynomial of $x$ and $y$ axes (Kuntschner et al 2009). Therefore, the row number at which the count rate of GU Psc b reaches the maximum in the $J^{\prime}$ and $H^{\prime}$ bands are slightly different.
}
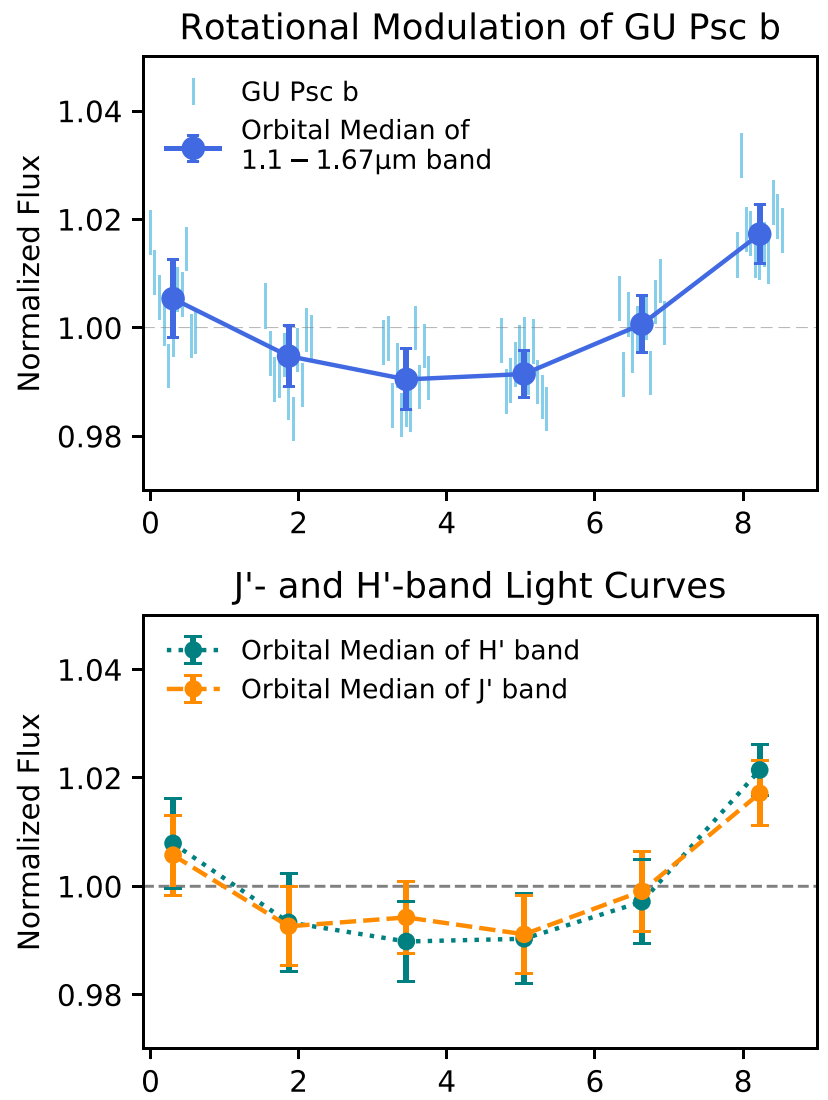

Light Curves of GU Psc b and Background Sources

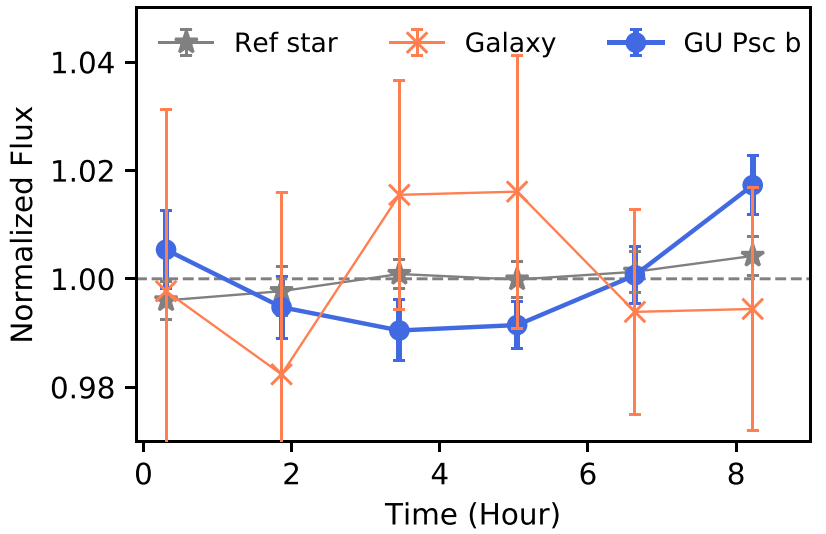

Figure 3. Top panel: the rotational modulation of GU Psc b shows a $2.7 \pm 0.8 \%$ peak-to-trough flux variation during the $8 \mathrm{hr}$ observation. In each $H S T$ orbit, we plot 11 single-exposure photometric points as light blue error bars and the orbital median as dark blue points. The error bar of the orbital median is the standard deviation of photometric points per HST orbit. Middle panel: the $J^{\prime}$ - and $H^{\prime}$-band light curves show similar light curve profiles. Bottom panel: the white light curve of GU Psc b is distinct from the flux variations of the nearby background sources.

GU Psc b. Our HST/G141 observations provide the first flux density measurements of GU Psc b in the water-band absorption region of $1.1-1.2 \mu \mathrm{m}$. After the $J$-band flux normalization of the two field T dwarfs' spectra, GU Psc b's spectrum better matches the T4.5 spectrum at wavelengths of $\lambda<1.3 \mu \mathrm{m}$ but better matches the T3 spectrum at longer wavelengths $(\lambda>1.3 \mu \mathrm{m})$.

In the bottom panel of Figure 2, we plot the binned ratio of the median spectra of the sixth and the third orbit, the orbits at which the broadband-integrated flux density reaches its 
maximum and minimum. The maximum/minimum flux ratio shows no strong wavelength-dependence for the rotational modulations, similar to that of T0 dwarf SIMP 0136 (Apai et al. 2013). After excluding the low signal-to-noise ratio water band $(1.37-1.47 \mu \mathrm{m})$, the mean maximum/minimum flux ratio is about $3.0 \%$. The fitted slope $\left(m=0.025 \pm 0.020 \mu \mathrm{m}^{-1}\right)$ for the maximum/minimum flux ratio suggests that there is no significant wavelength dependence.

\subsection{Light Curves of Rotational Modulation}

We plot the integrated white $(1.1-1.67 \mu \mathrm{m})$ light curves of GU Psc $b$ and those of the other two nearby sources in Figure 3. The light curve of GU Psc b manifests a sinusoidal profile with a period longer than the observation baseline. The sinusoidal pattern of GU Psc b's light curve is distinct from the almost flat light curve of the reference star and the choppy light curve of the nearby galaxy. The $J^{\prime}$-band $(1.18-1.33 \mu \mathrm{m})$ and $H^{\prime}$-band $(1.50-1.65 \mu \mathrm{m})$ light curves also show similar profiles as that of the integrated white light curve. Based on the integrated white light curve, the rotational modulation amplitude is at least $1.35 \%$, or $2.7 \pm 0.8 \%$ for the peak-to-trough flux variation (i.e., the ratio of the integrated flux median at the sixth orbit to that at the third orbit). This variability level is consistent with the previously reported marginal detection of peak-to-trough variability of $4 \% \pm 1 \%$ at a timescale of $\sim 6 \mathrm{hr}$ by Naud et al. (2017).

Because of the incomplete phase coverage, the fitted rotational period is degenerate with the amplitude for a sinusoidal model (see Appendix B). Therefore, we only place a lower limit of $8 \mathrm{hr}$ on the rotational period, corresponding to the baseline of the HST observations.

\section{Rotational Modulations on the Color-Magnitude Diagram}

Color-magnitude diagram (CMD) is a useful tool for studying the brown dwarf atmosphere evolution with thousands of brown dwarf photometric and parallax observations (e.g., Dupuy \& Liu 2012; Best et al. 2015). Meanwhile, an increasing number of brown dwarfs with detected rotational modulations through $H S T /$ G141 time-series spectral observations have been reported. We compile 11 brown dwarfs with published HST/ G141 spectral observations (2MASS J22282889-4310262, Buenzli et al. 2012; SIMP J013656.5+093347.3 and 2MASS J21392676+0220226, Apai et al. 2013; Luhman 16B Buenzli et al. 2015; 2MASS J15074769-1627386 and 2MASS J18212815+1414010, Yang et al. 2015; WISEP J004701.06 +680352.1, Lew et al. 2016; HN Peg B, Zhou et al. 2018; PSO J318.5338-22.8603, Biller et al. 2018; LP 26-75 B, Manjavacas et al. 2018; Ross458c, Manjavacas et al. 2019) to study the color dependence of rotational modulations across different spectral types in the Two Micron All Sky Survey (2MASS) $M_{\mathrm{J}}$ versus $J-H \mathrm{CMD}$. In this section, we describe the conversion from $H S T$ spectra to broadband photometry, the empirical models for color-magnitude variations, and discuss the overall trend in color-magnitude variations on CMD.

\subsection{Binning HST Time-series Spectra to Broadband Photometries}

The HST/G141 grism's wavelength coverage does not fully overlap with that of the 2MASS $H$ band. By weighting the $H S T /$ G141 spectral variations in the $H S T J^{\prime}(1.18-1.33 \mu \mathrm{m})$ and $H^{\prime}$
(1.50-1.65 $\mu \mathrm{m})$ bands with the 2MASS $J$-band and $H$-band spectral response curves (Wright et al. 2010), we implicitly assume that modulation amplitudes $\Delta J^{\prime}=2 \operatorname{MASS} \Delta J$ and $\Delta H^{\prime}=2$ MASS $\Delta H$.

To plot the $H S T /$ G141 spectral variations on a 2 MASS CMD, we also adopt the 2MASS $J$ - and $H$-band magnitudes (Cutri et al. 2003) as the mean magnitudes of the HST $J^{\prime}$ - and $H^{\prime}$-band modulations, except for the most variable object, $2 \mathrm{M} 2139 .{ }^{20}$ We first scale the $J$-band peak of the $0.6-2.65 \mu \mathrm{m}$ 2M2139 spectrum from the SpeX library (Burgasser 2014) to be the same as that of the averaged HST spectra. Then we use the scaled SpeX spectrum to calculate the mean magnitude in the 2MASS $J$ and $H$ bands during the HST observation of $2 \mathrm{M} 2139$.

The timescale of intrinsic color-magnitude variations from rotational modulations of brown dwarfs is typically on the order of hours. Meanwhile, the observed color-magnitude variations on several minute timescale are likely dominated by photon noise and/or systematics. To study the intrinsic colormagnitude variations across the rotational phase, for objects with long rotational periods $(P>5 \mathrm{hr})$ we bin the photometric points in the each $H S T$ orbit (total exposure time of 30-40 minutes) with the median value. We estimate the uncertainties of the median with the standard deviations of color and magnitude variations in each bin. These uncertainties are conservative estimates because they include the photon noise and the readout noise, variations from time-variable systematics (e.g., ramp effect), and intrinsic variability of objects. For objects with periods less than $5 \mathrm{hr}$, we choose not to bin the photometric points so that the cadence of the colormagnitude variations is less than $10 \%$ of the rotational phase.

\subsection{Empirical Models for Color-Magnitude Variations}

The $J-H$ color change due to rotational modulations in any individual object is much smaller compared to the $J-H$ color evolution across the $\mathrm{L} / \mathrm{T}$ transition. To visualize the small scale of color change and the large scale of color evolution in the CMD, we fit a straight line to the magnitude-color variation (i.e., $M_{\mathrm{J}}$ versus $J-H$ ) for the rotational modulations of each object. We then plot the fitted straight line of the magnitudecolor variation on the CMD. We obtain the best-fit slope and $y$ intercept using an orthogonal distance regression algorithm with scipy.odr (see also Figure 4), which minimizes the orthogonal distance, weighted by both $x$ - and $y$-axis uncertainties, between photometric points and the straight-line model. We use the covariance matrix of the fitted parameters to calculate the standard deviation of the fitted slope and $y$ intercept.

We note that this linear fit is only for illustrating the primary direction of the color changes with respect to the brightness variations. The color-magnitude variations could be nonlinear too. For example, the subpanel plots in Figure 5 suggest that a linear model may not fit well to the color-magnitude variations of GU Psc b and 2M2228. As one of the simple, periodic nonlinear models, we fit an ellipse to the color-magnitude variations and show the direction of the color change with the semimajor axis of the ellipse. The fitted color-magnitude changes of the ellipse are not unique (e.g., the elliptical and

\footnotetext{
${ }^{20}$ Even with the assumption of $\Delta J^{\prime}=2 \mathrm{MASS} \Delta J$, the mean of $H S T J^{\prime}(t)$ does not necessarily equal that of 2 MASS $J$, especially for an object with a large modulation amplitude.
} 


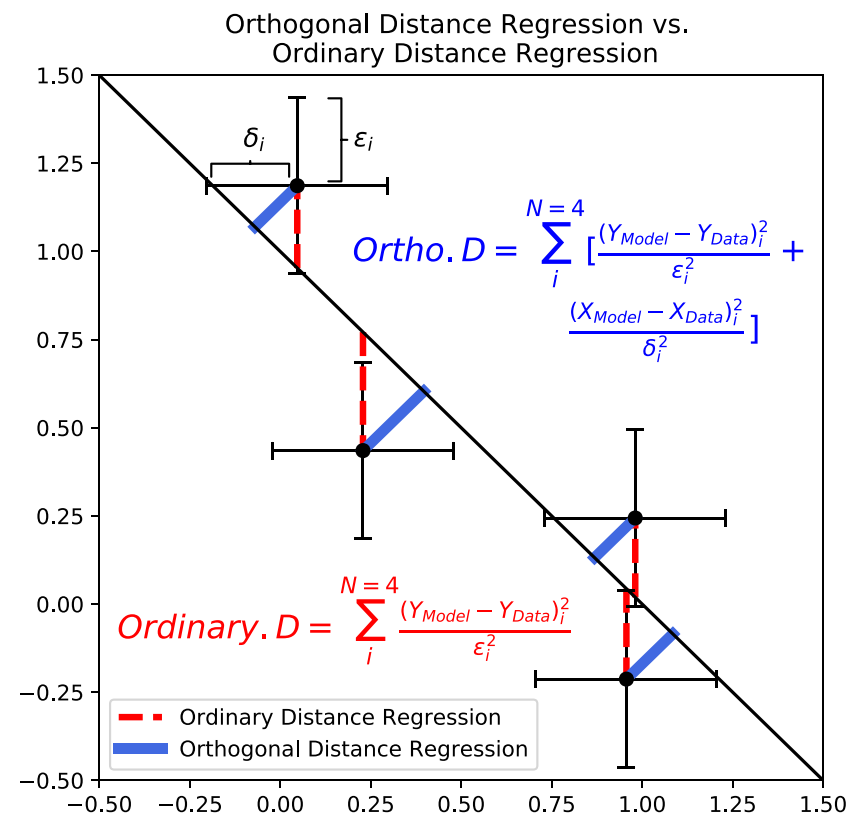

Figure 4. Graphic illustration of the difference between the ordinary distance least-squares regression and the orthogonal distance least-squares regression. In brief, the latter minimizes the uncertainty-weighted orthogonal distance between the data and model while the prior minimizes the uncertainty-weighted vertical distance between the data and model.

circular fit for GU Psc b in Figure 5) and are sensitive to the phase coverage of the light curves.

For reference, we also plot the color-magnitude variations of a blackbody with varying temperatures, either as hotter or cooler from that with the effective temperature for each object as a dashed gray line in the subpanels of Figure 5. In the nearIR $J$ and $H$ bands, a sum of blackbodies with different temperatures is still similar to a Planck function (Schwartz \& Cowan 2015). Therefore, this model acts as a toy model of a heterogeneous photosphere with a mixture of blackbodies at different temperatures.

\subsection{Result of Fitting Color-Magnitude Variations}

In the left panel of Figure 5, we present the trajectories of the rotational modulations of 12 ultracool atmospheres spanning a broad spectral type range. We plot the colors and magnitudes of field brown dwarfs whose with a signal-to-noise ratio $>10$ for $J$ - and $H$-band photometry from the updated catalog of Dupuy \& Liu (2012). The solid gray line is the empirically derived polynomial function of magnitude versus spectral type from Dupuy \& Liu (2012). In the $3 \times 4$ subpanels in Figure 5, we show the color-magnitude variations of individual objects and the model fitting results. We plot the linear fit and the blackbody models for every object. For GU Psc b and 2M2228 that demonstrate nonlinear color-magnitude variations, we also plot the best-fit ellipses for reference. For 2M2228, the nonlinearity of variations is more apparent with the HST orbital photometric points.

The slope of the fitted lines qualitatively shows how much the $J-H$ colors change with respect to the $J$-band flux variations. Among the plotted objects, 2M2139 demonstrates the largest color change $\left(\Delta\left(J^{\prime}-H^{\prime}\right)=0.0378 \pm 0.0015\right)$, but it is still much smaller than its $J^{\prime}$-band magnitude variations $\left(\Delta J^{\prime} \sim 0.300 \pm 0.001\right)$. All of the plotted objects show less change in their colors compared to their modulation amplitudes. In other words, all objects show only weak color changes in their rotational modulations.

In the CMD, a positive slope suggests that the object becomes brighter and redder, and a negative slope suggests that the object becomes brighter and bluer. Most of the objects show negative slopes, especially among L dwarfs. Among the plotted objects, only GU Psc b and 2M2228 show positive slopes. Our fitted slope uncertainty distribution suggests that there is about a $20 \%$ probability for GUPsc b to have a negative slope as seen in other L dwarfs. Therefore, the fitted slope of GU Psc b's color-magnitude variations is not significantly different from that of the other $\mathrm{L} / \mathrm{T}$ transition objects. There are two possible interpretations for the possible positive slope-either there is a phase shift between $J^{\prime}$ - and $H^{\prime}$-band light curves of GU Psc b, as in the case of $2 \mathrm{M} 2228$ (phase shift of $15^{\circ} \pm 2^{\circ}$; Buenzli et al. 2012), or the $H$-band modulation amplitude is indeed higher than that of the $J$ band. A longer baseline observation is needed to understand and verify if the color change of GU Psc b is indeed distinct from that of other L dwarfs.

\section{Discussions}

\subsection{The First Planetary-mass Object with Confirmed Modulations at the End of the L/T Transition}

The modulations of GU Psc b described here are the first in a planetary-mass object at the end of the L/T transition (T3-T5), confirming the previous $J$-band marginal detection by Naud et al. (2017). As the atmosphere evolves from $L$ to $T$ spectral type, the silicate cloud base presumably sank to deeper pressure (see the condensation curves in Helling \& Casewell 2014; Robinson \& Marley 2014), and hence the photosphere becomes less cloudy. Based on the observed rotational modulations, we argue that the photosphere of low-gravity mid-T dwarfs is not cloud free.

In addition to the modulations observed in GU Psc b, we have gathered a unique data set of rotational modulations found in planetary-mass objects across different effective temperatures, including the L7 spectral type PSOJ318 (Biller et al. 2015, 2018), T2 type SIMP0136 (Apai et al. 2013), and the T8 type Ross 458 c (Manjavacas et al. 2019). Our data set provides a useful reference point for future studies of cloud evolution across the $\mathrm{L} / \mathrm{T}$ transition. Furthermore, the wavelength dependence in rotational modulations of GUPsc b will be useful in testing predictions of different cloud models on the role of gravity in shaping cloud structure, as well as on testing the hypothesis that the $\mathrm{L} / \mathrm{T}$ transition, and maybe the $J$-band brightening too, is surface-gravity-dependent.

\subsection{The Modulation Amplitude and Rotational Period}

In any rotating atmosphere, an asymmetric brightness distribution leads to rotational modulations. The modulation amplitudes cannot be directly translated to brightness maps, as the hemisphere-integrated measurements necessarily result in information loss (although some inferences can be drawn, see Cowan \& Agol 2008; Apai et al. 2013; Cowan et al. 2013; Karalidi et al. 2016). The high modulation amplitude observed in GU Psc b suggests that there are prominent rotationally asymmetric features (dark or bright) that significantly $(>2 \%)$ impact even the hemisphere-integrated brightness of the photosphere. The features may be large and/or high contrast; if there are many features, these must be distributed 


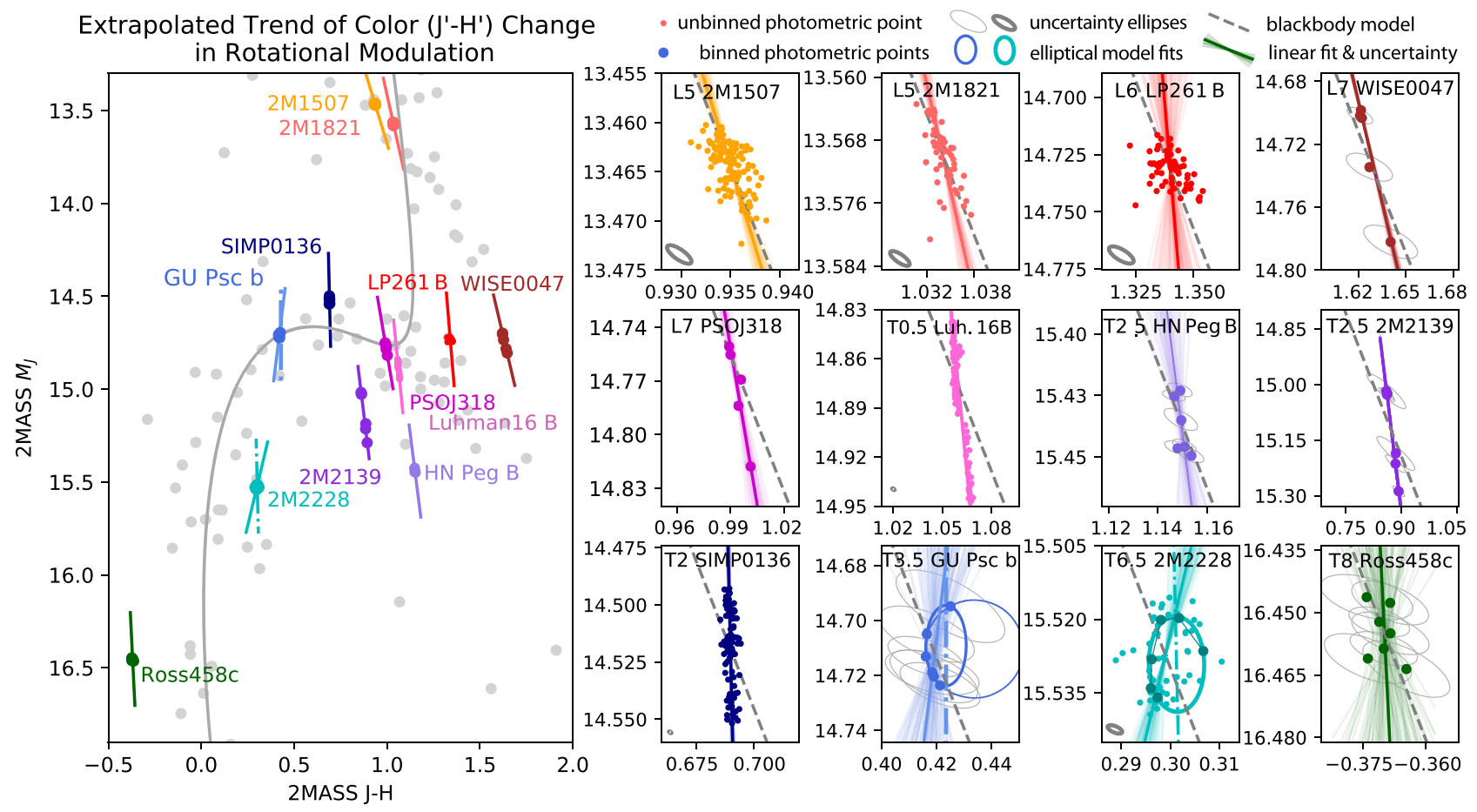

Figure 5. Left panel: the fitted linear trajectories of color-magnitude variations for 12 objects are plotted as solid lines. The almost vertical direction of the plotted trajectories suggests that most objects show relatively weak color changes compared to their rotational modulation amplitudes. Dashed-dotted lines are the semimajor axes of the fitted ellipses. The gray dots represent the colors and magnitudes of the brown dwarfs from Dupuy \& Liu (2012); the gray line is the empirically derived color-magnitude evolution curve from Dupuy \& Liu (2012). $3 \times 4$ panels on the right: zoomed-in plots for color-magnitude variations and the fitted slopes for individual objects. We plot the $H S T$ orbital photometric points (large solid dots) and the unbinned photometric points (small solid dots) for objects with long and short rotational periods, respectively (see Section 5.1). The uncertainty ellipses are plotted in gray. For clarity, we plot the typical uncertainty ellipses of single-exposure photometric points at the bottom left corner of the subpanels. Three models to fit the color-magnitude variations are plotted: a straight line (solid straight lines), ellipse (for 2M2228 and GU Psc b, curved solid lines), and blackbody with varying temperature (gray dashed lines). See Section 5 for more details on the models. The uncertainties of the linear models are plotted in semitransparent color lines. The semimajor axes of fitted ellipses are plotted in dashed-dotted lines. The left and right panels share the same aspect ratio.

asymmetrically. From IR rotational modulation surveys that include both high- and low-gravity brown dwarfs, we already know that brown dwarf atmospheres are ubiquitously heterogeneous (e.g., Buenzli et al. 2014; Metchev et al. 2015). Typical field brown dwarfs outside the $\mathrm{L} / \mathrm{T}$ spectral type transition have peak-to-trough flux variations of less than a $2 \%$ level in the $J$ band (Buenzli et al. 2014; Radigan et al. 2014). The observed peak-to-trough flux variations of $\sim 2.7 \%$ of GU Psc b is consistent with the conclusion of Radigan et al. (2014) that L/T transition objects are likely to have the largest modulation amplitudes. The high modulation amplitude of this low-gravity object provides another data point to test whether high modulation amplitude is correlated with low gravity, as claimed by Vos et al. (2017) based on a compilation of published variability amplitudes of brown dwarfs. The minimum period of $8 \mathrm{hr}$ is in line with the measured timescale of periodic modulations of other brown dwarfs, ranging from as short as $1.4 \mathrm{hr}$ (Buenzli et al. 2012) to $18 \mathrm{hr}$ or longer (e.g., 2M2148; Metchev et al. 2015). The actual rotational modulation profile may evolve with time, as seen in long-baseline observations of multiple $\mathrm{L} / \mathrm{T}$ transition brown dwarfs (e.g., Apai et al. 2017), most prominently detected in all three brown dwarfs (2M2139, 2M1324, and SIMP0136) monitored by the Spitzer Space Telescope in the Extrasolar Storms program (Yang et al. 2016; Apai et al. 2017). That study shows that the light curve evolution is the likely result of planetary-scale waves that modulated surface brightness (Apai et al. 2017), possibly through the interplay of atmospheric circulations, condensations, and cloud formation/dispersal
(Tan \& Showman 2017, 2019; Showman et al. 2019). These mechanisms may also be present in GUPsc b and their presence could be revealed by continuous observations over 3-4 rotational periods.

The lower limit of the rotational period of GU Psc b also provides another data point for studying the evolution of spin as a function of mass and age. Assuming that the radius of GU Psc b is roughly 1.3-1.4 Jupiter radius, as expected for a $100 \mathrm{Myr}$ old and $10-12 M_{\text {Jup }}$ object (Chabrier et al. 2000), with a minimum rotational period of $8 \mathrm{hr}$, the spin velocity is about $19.6-21 \mathrm{kms}^{-1}$. A longer period $(P>8 \mathrm{hr})$ will result in a slower spin. At the age of ABDMG, which is $\sim 120-200 \mathrm{Myr}$, our upper limit of the spin rate of GU Psc b is similar with that of other planetary-mass companions at different ages (3-300 Myr old; e.g., Snellen et al. 2014; Zhou et al. 2016; Biller et al. 2018; Bryan et al. 2018). Because the radii contract along with the loss of interior entropy for young objects, the spin velocities increase as the objects cool with age under the conservation of angular momentum. Therefore, the spin rates of GU Psc b could reach as high as $30 \mathrm{kms}^{-1}$ after radius contracting to one Jupiter radius. A better period constraint is required to test if the spin rate is consistent with the suggested universal spin-mass relation of Scholz et al. (2018; see also Zhou et al. 2019).

\subsection{Gray Modulations and Atmospheric Heterogeneity}

Both the color-magnitude variations shown in Figure 5 and the ratio of the brightest-to-dimmest spectra shown in Figure 2 
demonstrate that the modulation amplitudes of GUPsc b are similar in the $J^{\prime}$ and $H^{\prime}$ bands (peak-to-trough variation of $2.6 \% \pm 0.9 \%$ and $3.2 \% \pm 0.9 \%$, respectively), and hence mostly gray. Previous observations find similar modulation amplitudes in the $J$ and $H$ bands for $\mathrm{L} / \mathrm{T}$ transition objects (e.g., Artigau et al. 2009; Radigan et al. 2012; Apai et al. 2013), although the modulation amplitudes can be different in molecular bands (e.g., water) or at longer wavelengths (e.g., $\mathrm{K}_{\mathrm{s}}$ band; e.g., Apai et al. 2013; Biller et al. 2013). The modeling of these data suggest that spatial variations of cloud properties are responsible for the modulations (e.g., Apai et al. 2013). Detailed atmospheric modeling of space-based, highprecision time-domain data argues for simultaneous changes in cloud thickness and temperature as the cause for spatially varying cloud brightness (thin warm and thick cold clouds; Apai et al. 2013; Buenzli et al. 2015). GU Psc b also shows similarly gray rotational modulations. Therefore, we argue that correlated variations of cloud opacity and temperature are the most likely the cause of the observed gray modulations in GU Psc b.

The observed weak (non-zero) wavelength dependence may also carry information about spatial variation in the particle size distribution. A weak and positive wavelength dependence in rotational modulations could be explained by the varying presence of particles with sizes larger than one micron (e.g., see Figure 5 of Hiranaka et al. 2016). If this is true, then GU Psc b's atmosphere is in contrast to L dwarf atmospheres, which are often found to be reddened or extinguished by submicron grains (Hiranaka et al. 2016; Marocco et al. 2014; see also a retrieval analysis from Burningham et al. 2017). This would be consistent with the cloud thinning scenario that predicts larger mean particle sizes (or larger $f_{\text {sed }}$ ) for mid-T and smaller mean particle sizes (or smaller $f_{\text {sed }}$ ) for $\mathrm{L}$ dwarfs (Saumon \& Marley 2008).

\subsection{No Strong Color Change in Rotational Modulations Across the L/T Transition}

In the color-magnitude plot shown in Figure 5 even the most highly variable object (2M2139) shows only less than $20 \%$ relative difference between its $J$-band and the $H$-band modulation amplitudes $(31.4 \pm 0.1 \%$ versus $27.6 \pm 0.1 \%)$. All other objects show similarly gray color change in modulations $\left(\Delta\left(J^{\prime}-H^{\prime}\right) \ll \Delta J^{\prime}\right)$ : no known source is varying its color at a level comparable to the brightness modulations. In other words, the observed modulations in all sources are close to gray, albeit with some variety. The lack of strong color in rotational modulations in brown dwarfs with spectral types ranging from mid-L to late- $\mathrm{T}$ is consistent with the paradigm in which spatially heterogeneous clouds modulate the hemisphere-integrated brightness.

Given that the color changes due to atmospheric heterogeneity are different from the overall red-to-blue color evolution found across the $\mathrm{L} / \mathrm{T}$ transition (a large color evolution with only a small change in absolute $J$-band magnitude from L8-to-T5 spectral type), we conclude that atmospheric heterogeneity alone does not directly cause the drastic color evolution across the $\mathrm{L} / \mathrm{T}$ transition, at least for low-gravity atmospheres (i.e., GU Psc b, SIMP0136, PSOJ318). However, as previously suggested by Radigan et al. (2012), atmospheric heterogeneity could still affect the evolution of atmospheres over evolutionary timescales-an atmosphere with thinner clouds or more patchy cloud distribution cools more efficiently because more flux can be radiated from below the cloud base. More efficient cooling leads to a larger loss of entropy over evolutionary timescales. As a result, the loss of interior entropy is coupled with varying degrees of atmospheric heterogeneity and could still lead to the observed drastic color evolution in the $\mathrm{L} / \mathrm{T}$ transition. Our observations only probe the impact of atmospheric heterogeneity with fixed interior entropy in rotational timescales. Change of cloud structure will affect the relative abundance of objects with different spectral subtypes across the L/T transition, as predicted by Saumon \& Marley (2008). Observational-bias-corrected samples of brown dwarfs will be powerful to test the coupled evolution of the cloud structure and interior entropy over evolutionary timescales.

It is tempting to perceive a possible trend in Figure 5 in the slope of the magnitude-color variations from L to T dwarfs: $\mathrm{L}$ dwarfs become brighter and bluer, early-T dwarfs become brighter with almost no color change, and the two out of three mid-to-late $\mathrm{T}$ dwarfs become brighter and redder. However, we are limited by the small sample size of $\mathrm{T}$ dwarfs with timeresolved spectrophotometry, and the uncertain slope of GU Psc b due to incomplete phase coverage. Therefore, we remain cautious about the significance of this tentative trend. More long-term, time-resolved spectroscopy of $\mathrm{T}$ dwarfs is needed to verify this tentative trend and to test if there is a statistically significant difference between $\mathrm{L}$ and $\mathrm{T}$ dwarfs in the nature of their wavelength-dependent rotational modulations.

\section{Conclusions}

In this study we present the HST time-resolved near-IR spectral variations of the planetary-mass, T3.5 spectral type object GU Psc b. The key conclusions of our study are as follows.

1. We confirm the previously reported (Naud et al. 2017) tentative rotational modulations in the planetary-mass companion GU Psc b. This is the first planetary-mass object in the T3-T5 spectral range with confirmed rotational modulations.

2. Based on our HST WFC3/G141 observations we place a lower limit of $2.7 \pm 0.8 \%$ of the peak-to-trough flux variation of GU Psc b and a period of $8 \mathrm{hr}$ or longer. As our phase coverage is incomplete, it is likely that the actual flux variations are somewhat higher.

3 . We find mostly gray (wavelength-dependent slope of $m=0.025 \pm 0.020 \mu \mathrm{m}^{-1}$; see Figure 2) rotational modulations for wavelengths from 1.1 to $1.67 \mu \mathrm{m}$ excluding the water band. Based on the gray modulations, we argue that cloud opacity likely dominates the rotational modulations in the photosphere of low-gravity mid-T dwarf GU Psc b.

4. From our compilation of mid-L to late-T dwarfs, we find their rotational modulations to be mostly gray, including objects across the $\mathrm{L} / \mathrm{T}$ transition. We argue that atmospheric heterogeneity cannot explain the drastic color evolution across the $\mathrm{L} / \mathrm{T}$ transition over rotational timescales. Cloud heterogeneity could still play an important role in atmospheric evolution in the $\mathrm{L} / \mathrm{T}$ transition over evolutionary timescales.

5. From $\mathrm{L}$ to $\mathrm{T}$ dwarfs we find an interesting but tentative trend in the slope of the magnitude-color variations. If 
confirmed, this trend would indicate that the nature of the rotational modulation is spectral-type-dependent. However, more samples of the rotational modulations of $\mathrm{T}$ dwarfs with complete phase coverage are needed to test the significance of the trend.

Together with 2M1207b, 2MASS J13243553+6358281, PSOJ318, Ross $458 \mathrm{c}$, and SIMP0136, GU Psc b is another rare planetary-mass objects with a large IR modulation amplitude. This object is yet a unique example of a T3-T5 spectral type, low-gravity object with detected rotational modulations. As such it provides an important reference to study cloud structure evolution as functions of effective temperature and gravity. Soon the higher sensitivity and wider wavelength coverage of next-generation telescopes such as the James Webb Space Telescope (JWST) and Extremely Large Telescopes (Giant Magellan Telescope, Thirty Meter Telescope, and the European ELT) will transform time-resolved spectroscopy into an even more powerful method for constraining the cloud structure and particle size distribution.

We would like to thank the anonymous referee for constructive comments and suggestions that significantly improve this paper. We would like to thank Esther Buenzli for providing the time-resolved spectra of Luhman 16b. Support for program number HST-GO-14241 was provided by NASA through a grant from the Space Telescope Science Institute, which is operated by the Association of Universities for Research in Astronomy, Incorporated, under NASA contract NAS5-26555. This research has benefited from the Montreal Brown Dwarf and Exoplanet Spectral Library, maintained by Jonathan Gagné and the SpeX Prism Spectral Libraries, maintained by Adam Burgasser at http://pono.ucsd. edu/ adam/browndwarfs/spexprism. This publication makes use of data products from the Two Micron All Sky Survey, which is a joint project of the University of Massachusetts and the Infrared Processing and Analysis Center/California Institute of Technology, funded by the National Aeronautics and Space Administration and the National Science Foundation. This work has made use of data from the European Space Agency (ESA) mission Gaia (https://www.cosmos.esa.int/ gaia), processed by the Gaia Data Processing and Analysis Consortium (DPAC, https://www.cosmos.esa.int/web/gaia/ dpac/consortium). Funding for DPAC has been provided by national institutions, in particular the institutions participating in the Gaia Multilateral Agreement.

\section{Appendix A Contamination Model}

To provide a quantitative estimate of the contamination, we use a model with three 1D Moffat profile to fit the horizontally summed (sum of pixels of columns 440-570 in Figure 1(b), corresponding to the $1.1-1.7 \mu \mathrm{m}$ region of GUPscb's spectrum) pixel count rates of the GU Psc b, the nearby galaxy, and the reference star. The reduced chi-square from the model

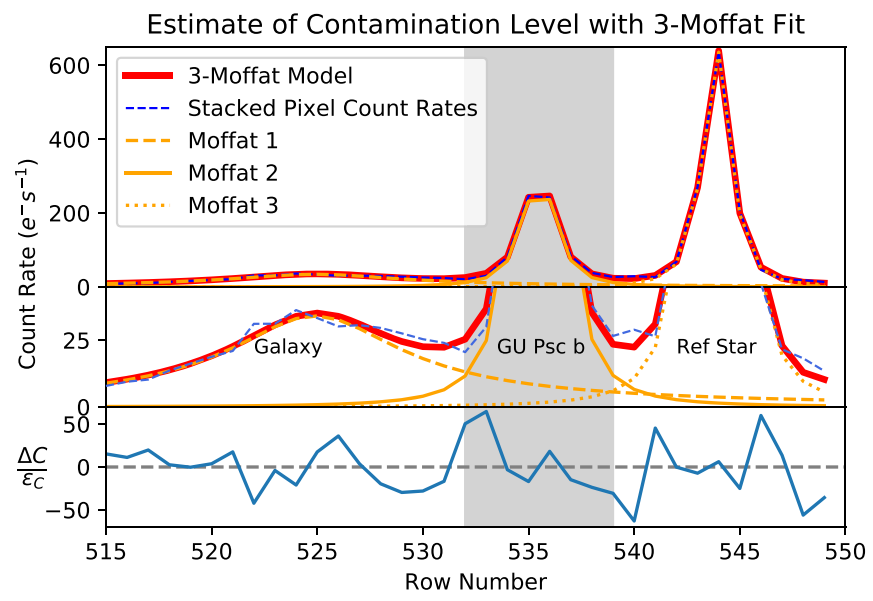

Figure A1. Top: the fitting result of the three-Moffat model (solid red line) to the horizontally summed (i.e., sum of pixel count rates from columns 440-570 in Figure 1) pixel count rates (dashed blue line). The three-Moffat profiles are plotted in orange dashed, dotted, and solid lines. Middle panel: same as the top panel with a zoomed-in view of the fitting result for the Galaxy. Bottom panel: the residual between the model and measured count rate $\Delta C$ in units of the observation noise $\epsilon_{c}$ (photon and readout noise).

fitting is large $(\sim 700)$ because of the significant deviation at the wing. Based on the best-fit Moffat profiles as shown in Figure A1, the galaxy's and the reference star's flux in the 8 pixel wide shaded region is about $10 \%$ and $3 \%$ of the GU Psc b's flux, respectively. Contamination levels in the $J^{\prime}$ and $H^{\prime}$ bands are thus lower than $13 \%$ because GU Psc b's spectral intensities are higher in these bands than the averaged intensity over 1.1-1.7 $\mu \mathrm{m}$. As mentioned in Section 3.1, the low contamination level $(<13 \%)$ and the measured variability of the galaxy and reference star together suggests that the detected flux variation of GU Psc b is intrinsic.

\section{Appendix B \\ Posterior Distribution of Rotational Period with Markov Chain Monte Carlo method}

We construct a sinusoidal model on top of a linear slope, which represents the possible visit-long HST systematic (e.g., Section 3.1), for calculating the log-likelihood function of the MCMC method with emcee. We adopt the log-uniform priors with a period of $P=[5,50 \mathrm{hr}]$, phase $=[0,2 \pi]$, baseline $c=[0.5,1.5]$, amplitude $=[0.003,1.2]$, and slope $=\left[10^{-5}\right.$, $\left.2 \times 10^{-3} \mathrm{hr}^{-1}\right]$. We then run MCMC with 50 walkers for 500,000 steps and plot the posterior distribution in Figure B1. We note that the upper bound (50 hours) of the posterior distribution of period is equivalent to the upper bound of prior. Therefore, the upper bounds of the period, amplitude, and baseline are unconstrained based on the MCMC result. The posterior distribution suggests that the rotational period is degenerate with the variability amplitude, phase, and with the baseline. The marginalized posterior distribution of the slope 


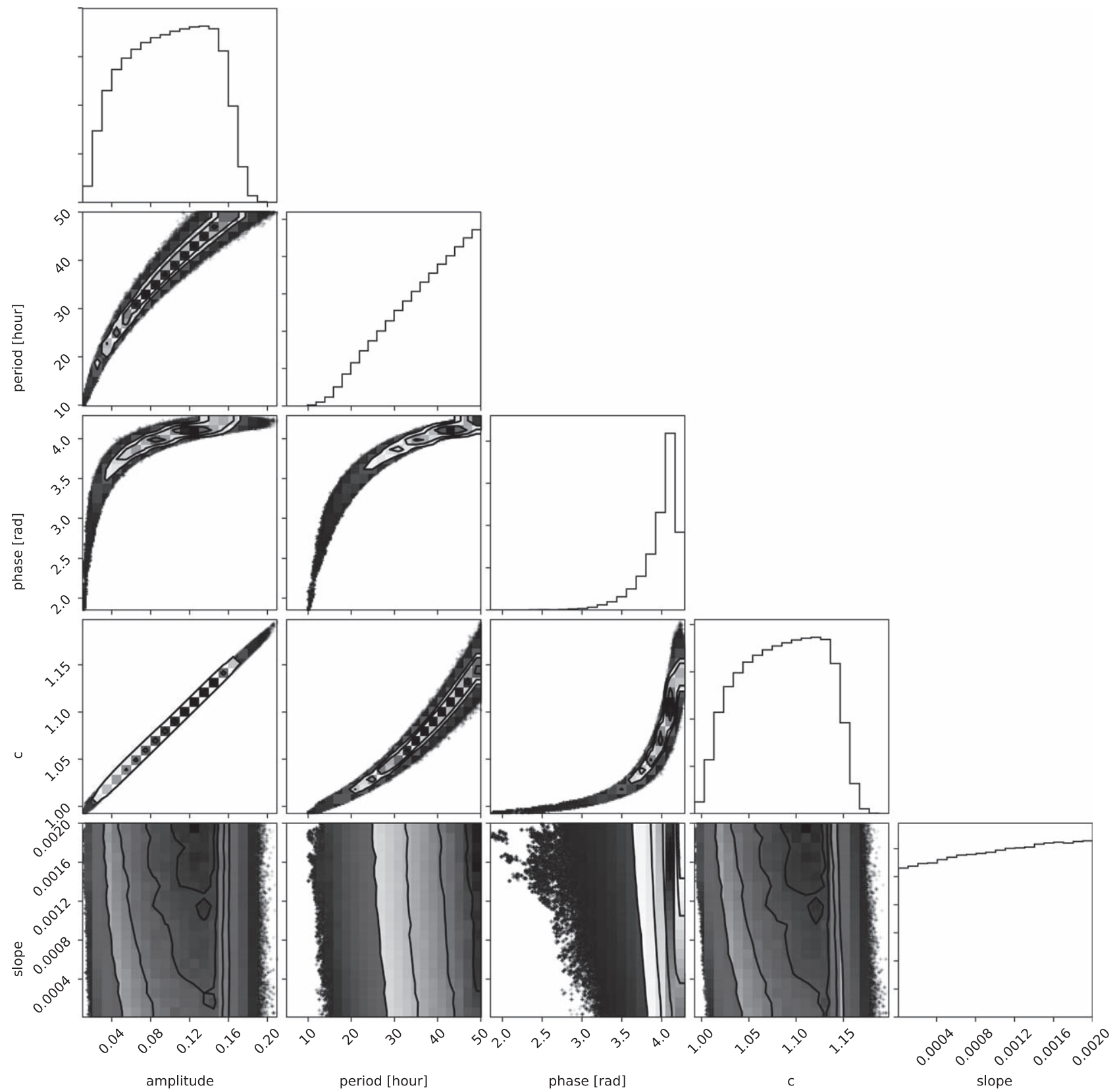

Figure B1. Posterior distribution results from the Markov Chain Monte Carlo (MCMC) method for a sine wave model on top of a linear slope. The posterior distribution of the rotational period is degenerate with other model parameters.

shows that including the possible visit-long $H S T$ systematic as a free parameter does not affect the result.

\section{ORCID iDs}

Ben W. P. Lew (ii) https://orcid.org/0000-0003-1487-6452 Dániel Apai (1) https://orcid.org/0000-0003-3714-5855 Yifan Zhou (ㄴ) https://orcid.org/0000-0003-2969-6040 Jacqueline Radigan (ib https://orcid.org/0000-0002-6732-3651 Mark Marley (i) https://orcid.org/0000-0002-5251-2943 Glenn Schneider (10) https://orcid.org/0000-0002-4511-5966 Nicolas B. Cowan (1) https://orcid.org/0000-0001-6129-5699 Paulo A. Miles-Páez i https://orcid.org/0000-0003-2446-8882 Elena Manjavacas (1) https://orcid.org/0000-0003-0192-6887 Theodora Karalidi (i) https://orcid.org/0000-0001-7356-6652
Patrick J. Lowrance iㅣ https://orcid.org/0000-0001-8014-0270 Adam J. Burgasser (i) https://orcid.org/0000-0002-6523-9536

\section{References}

Ackerman, A. S., \& Marley, M. S. 2001, ApJ, 556, 872

Apai, D., Karalidi, T., Marley, M. S., et al. 2017, Sci, 357, 683

Apai, D., Radigan, J., Buenzli, E., et al. 2013, ApJ, 768, 121

Artigau, E. 2018, in Handbook of Exoplanets, ed. H. J. Hans \& J. A. Belmonte (Cham: Springer), 94

Baraffe, I., Chabrier, G., Barman, T. S., Allard, F., \& Hauschildt, P. H. 2003, A\&A, 402, 701

Barrado y Navascués, D., \& Martín, E. L. 2003, AJ, 126, 2997

Berta, Z. K., Charbonneau, D., Désert, J.-M., et al. 2012, ApJ, 747, 35

Bertin, E., \& Arnouts, S. 1996, A\&AS, 117, 393

Best, W. M. J., Liu, M. C., Magnier, E. A., et al. 2015, ApJ, 814, 118 
Biller, B. 2017, AstRv, 13, 1

Biller, B. A., Crossfield, I. J. M., Mancini, L., et al. 2013, ApJL, 778, L10

Biller, B. A., Vos, J., Bonavita, M., et al. 2015, ApJL, 813, L23

Biller, B. A., Vos, J., Buenzli, E., et al. 2018, AJ, 155, 95

Artigau, É, Bouchard, S., Doyon, R., \& Lafrenière, D. 2009, ApJ, 701, 1534

Bowler, B. P., Liu, M. C., Shkolnik, E. L., \& Dupuy, T. J. 2013, ApJ, 774, 55

Gaia Collaboration, Brown, A. G. A., Vallenari, A., et al. 2018, A\&A, 616, A1

Bryan, M. L., Benneke, B., Knutson, H. A., Batygin, K., \& Bowler, B. P. 2018, NatAs, 2, 138

Buenzli, E., Apai, D., Morley, C. V., et al. 2012, ApJL, 760, L31

Buenzli, E., Apai, D., Radigan, J., Reid, I. N., \& Flateau, D. 2014, ApJ, 782, 77

Buenzli, E., Saumon, D., Marley, M. S., et al. 2015, ApJ, 798, 127

Burgasser, A. J. 2014, in ASI Conf. Ser. 11, International Workshop on Stellar Spectral Libraries, ed. H. P. Singh, P. Prugniel, \& I. Vauglin (Bengaluru: ASI), 7

Burgasser, A. J., Geballe, T. R., Leggett, S. K., Kirkpatrick, J. D., \& Golimowski, D. A. 2006a, ApJ, 637, 1067

Burgasser, A. J., Kirkpatrick, J. D., Cruz, K. L., et al. 2006b, ApJS, 166, 585

Burgasser, A. J., Marley, M. S., Ackerman, A. S., et al. 2002, ApJL, 571, L151

Burgasser, A. J., McElwain, M. W., Kirkpatrick, J. D., et al. 2004, AJ, 127, 2856

Burningham, B., Marley, M. S., Line, M. R., et al. 2017, MNRAS, 470, 1177

Burrows, A., Sudarsky, D., \& Hubeny, I. 2006, ApJ, 640, 1063

Chabrier, G., Baraffe, I., Allard, F., \& Hauschildt, P. 2000, ApJ, 542, 464

Charnay, B., Bézard, B., Baudino, J.-L., et al. 2018, ApJ, 854, 172

Cowan, N. B., \& Agol, E. 2008, ApJL, 678, L129

Cowan, N. B., Fuentes, P. A., \& Haggard, H. M. 2013, MNRAS, 434, 2465

Cutri, R. M., Skrutskie, M. F., van Dyk, S., et al. 2003, yCat, II/246, 0

Dupuy, T. J., \& Liu, M. C. 2012, ApJS, 201, 19

Dupuy, T. J., Liu, M. C., \& Ireland, M. J. 2009, ApJ, 699, 168

Gaia Collaboration, Prusti, T., de Bruijne, J. H. J., et al. 2016, A\&A, 595, A1

Helling, C., \& Casewell, S. 2014, A\&ARv, 22, 80

Hiranaka, K., Cruz, K. L., Douglas, S. T., Marley, M. S., \& Baldassare, V. F. 2016, arXiv:1606.09485

Karalidi, T., Apai, D., Marley, M. S., \& Buenzli, E. 2016, ApJ, 825, 90

Kümmel, M., Kuntschner, H., Walsh, J. R., \& Bushouse, H. 2011, Master Sky Images for the WFC3 G102 and G141 Grisms, ST-ECF Instrument Science Report, WFC3-2011-01

Kümmel, M., Walsh, J. R., Pirzkal, N., Kuntschner, H., \& Pasquali, A. 2009, PASP, 121, 59

Kuntschner, H., Bushouse, H., Kümmel, M., \& Walsh, J. R. 2009, WFC3 SMOV Proposal 11552: Calibration of the G141 Grism, ST-ECF Instrument Science Report, WFC3-2009-17

Kuntschner, H., Kümmel, M., Walsh, J. R., \& Bushouse, H. 2011, Revised Flux Calibration of the WFC3 G102 and G141 Grisms, ST-ECF Instrument Science Report, WFC3-2011-05

Lew, B. W. P., Apai, D., Zhou, Y., et al. 2016, ApJL, 829, L32

Liu, M. C., Dupuy, T. J., \& Allers, K. N. 2016, ApJ, 833, 96

Long, K. S., Baggett, S. M., MacKenty, J. W., \& McCullough, P. M. 2014, Attempts to Mitigate Trapping Effects in Scanned Grism Observations of Exoplanet Transits with WFC3/IR, WFC3 Instrument Science Report, 2014-14 Luri, X., Brown, A. G. A., Sarro, L. M., et al. 2018, A\&A, 616, A9
Malo, L., Doyon, R., Lafrenière, D., et al. 2013, ApJ, 762, 88 Manjavacas, E., Apai, D., Lew, B. W. P., et al. 2019, ApJL, 875, L15 Manjavacas, E., Apai, D., Zhou, Y., et al. 2018, AJ, 155, 11

Mann, A. W., Brewer, J. M., Gaidos, E., Lépine, S., \& Hilton, E. J. 2013, AJ, 145,52

Marley, M. S., Saumon, D., Cushing, M., et al. 2012, ApJ, 754, 135

Marley, M. S., Saumon, D., \& Goldblatt, C. 2010, ApJL, 723, L117

Marocco, F., Day-Jones, A. C., Lucas, P. W., et al. 2014, MNRAS, 439, 372

Metchev, S. A., Heinze, A., Apai, D., et al. 2015, ApJ, 799, 154

Metchev, S. A., \& Hillenbrand, L. A. 2006, ApJ, 651, 1166

Miles-Páez, P. A., Metchev, S., Apai, D., et al. 2019, ApJ, 883, 181

Miles-Páez, P. A., Metchev, S., Luhman, K. L., Marengo, M., \& Hulsebus, A. 2017, AJ, 154, 262

Naud, M.-E., Artigau, É., Malo, L., et al. 2014, ApJ, 787, 5

Naud, M.-E., Artigau, É., Rowe, J. F., et al. 2017, AJ, 154, 138

Newton, E. R., Charbonneau, D., Irwin, J., et al. 2014, AJ, 147, 20

Norton, A. J., Wheatley, P. J., West, R. G., et al. 2007, A\&A, 467, 785

Radigan, J. 2014, ApJ, 797, 120

Radigan, J., Jayawardhana, R., Lafrenière, D., et al. 2012, ApJ, 750, 105

Radigan, J., Lafrenière, D., Jayawardhana, R., \& Artigau, E. 2014, ApJ, 793, 75

Riaz, B., Gizis, J. E., \& Harvin, J. 2006, AJ, 132, 866

Robinson, T. D., \& Marley, M. S. 2014, ApJ, 785, 158

Saumon, D., \& Marley, M. S. 2008, ApJ, 689, 1327

Schlawin, E., Burgasser, A. J., Karalidi, T., Gizis, J. E., \& Teske, J. 2017, ApJ, 849,163

Scholz, A., Moore, K., Jayawardhana, R., et al. 2018, ApJ, 859, 153

Schwartz, J. C., \& Cowan, N. B. 2015, MNRAS, 449, 4192

Showman, A. P., Tan, X., \& Zhang, X. 2019, ApJ, 883, 4

Smith, R. M., Zavodny, M., Rahmer, G., \& Bonati, M. 2008, Proc. SPIE, 7021, $70210 \mathrm{~K}$

Snellen, I. A. G., Brandl, B. R., de Kok, R. J., et al. 2014, Natur, 509, 63

Tan, X., \& Showman, A. P. 2017, ApJ, 835, 186

Tan, X., \& Showman, A. P. 2019, ApJ, 874, 111

Tremblin, P., Amundsen, D. S., Chabrier, G., et al. 2016, ApJL, 817, L19

Tremblin, P., Padioleau, T., Phillips, M. W., et al. 2019, ApJ, 876, 144

Tsuji, T., \& Nakajima, T. 2003, ApJL, 585, L151

Vos, J. M., Allers, K. N., \& Biller, B. A. 2017, ApJ, 842, 78

Wakeford, H. R., Sing, D. K., Evans, T., Deming, D., \& Mandell, A. 2016 , ApJ, 819, 10

West, A. A., Hawley, S. L., Bochanski, J. J., et al. 2008, AJ, 135, 785

White, R. J., \& Basri, G. 2003, ApJ, 582, 1109

Wright, E. L., Eisenhardt, P. R. M., Mainzer, A. K., et al. 2010, AJ, 140, 1868

Yang, H., Apai, D., Marley, M. S., et al. 2015, ApJL, 798, L13

Yang, H., Apai, D., Marley, M. S., et al. 2016, ApJ, 826, 8

Zhou, Y., Apai, D., Lew, B. W. P., et al. 2019, AJ, 157, 128

Zhou, Y., Apai, D., Lew, B. W. P., \& Schneider, G. 2017, AJ, 153, 243

Zhou, Y., Apai, D., Metchev, S., et al. 2018, AJ, 155, 132

Zhou, Y., Apai, D., Schneider, G. H., Marley, M. S., \& Showman, A. P. 2016, ApJ, 818, 176

Zuckerman, B., Song, I., \& Bessell, M. S. 2004, ApJL, 613, L65 